\title{
TENDÊNCIAS EMPREENDEDORAS DOS ENFERMEIROS DE UM HOSPITAL UNIVERSITÁRIO
}

Fabiana Gallo COSTA a , Helena Heidtmann VAGHETTI ${ }^{\mathrm{b}}$,

Daniela Faustino Gonçalves MARTINELLO ${ }^{c}$, Daniel Pinho MENDES ${ }^{\mathrm{d}}$, Alessandra Chaves TERRA ${ }^{\text {e }}$, Simone Quadros ALVAREZ ${ }^{\text {f }}$ Luiz Augusto Pinto LEMOSg

\section{RESUMO}

Estudo quantitativo que objetivou identificar tendências empreendedoras dos enfermeiros de um hospital universitário e relacioná-las com idade, tempo de trabalho e conclusão do curso de enfermagem. Estudo quantitativo, transversal contemporâneo realizado em 2010. Todos os 60 enfermeiros responderam ao questionário "Tendência Empreendedora Geral", com cinco categorias. Na categoria "criatividade", um enfermeiro obteve dois pontos, em "necessidade de realização”, um enfermeiro somou 12 pontos; em “impulso e determinação”, quatro enfermeiros alcançaram maior pontuação; em "riscos calculados/moderados", a maior pontuação foi 10 pontos; em "autonomia/independência”, nove enfermeiras obtiveram um ponto. Idades entre 27 e 33 anos mostraram maior tendência empreendedora. Existe decréscimo da tendência empreendedora dos enfermeiros com idades superiores a 43 até 56 anos, com mais de 17 anos de conclusão de curso e com maior tempo de trabalho. São necessárias ações que estimulem enfermeiros na faixa etária em declínio da tendência empreendedora e aqueles com maior tempo de formação e trabalho.

Descritores: Enfermagem. Recursos humanos de enfermagem no hospital. Inovação organizacional.

\section{RESUMEN}

Estudio cuantitativo que buscó identificar las tendencias emprendedoras de enfermeros de un hospital universitario y relacionarlas con la edad, tiempo de trabajo en el hospital y tiempo de la finalización del curso de enfermería. Estudio cuantitativo, transversal contemporáneo realizado en 2010. Todos los 60 enfermeros del hospital respondieron al cuestionario de Tendencia Emprendedora General con cinco categorías. En la categoría de creatividad, un enfermero obtiene dos puntos, en la necesidad de realización un enfermero totalizó 12 puntos; en el impulso y la determinación cuatro enfermeros lograron mayor puntuación; en los riesgos calculados/moderados la mayor puntuación fue de 10 puntos y la más baja un punto; en la autonomíal independencia nueve enfermeros obtuvieron un punto. Edades entre 27 y 33 mostraron las tendencias más emprendedoras. Hay disminución de esas tendencias en de los enfermeros mayores de 43 y ate 56 años, para aquellos que se graduaron hace más de 17 años y aquellos con más tiempo de trabajo en el hospital. Son importantes actividades que estimulen a los enfermeros que se encuentran en el intervalo de edad de disminución de la tendencia emprendedora, así como los que tienen más tiempo de formación e trabajo en el hospital.

Descriptores: Enfermería. Personal de enfermería en hospital. Innovación organizacional.

Título: Tendencias de actitudes emprendedoras de los enfermeros en un hospital universitario.

a Enfermeira graduada pela Universidade Federal do Rio Grande (FURG). Rio Grande, Rio Grande do Sul, Brasil. b Doutora em Enfermagem. Docente da Escola de Enfermagem, FURG. Rio Grande, Rio Grande do Sul, Brasil.

c Mestre em Enfermagem pela FURG. Rio Grande, Rio Grande do Sul, Brasil.

d Mestrando do Programa de Pós-Graduação em Enfermagem da FURG. Rio Grande, Rio Grande do Sul, Brasil.

e Mestre em Enfermagem pela FURG. Rio Grande, Rio Grande do Sul, Brasil.

f Mestre em Enfermagem pela FURG. Rio Grande, Rio Grande do Sul, Brasil.

g Mestre em Engenharia. Docente do Instituto de Matemática, Estatística e Física, FURG. Rio Grande, Rio Grande do Sul, Brasil.

Versão on-line em Português/Inglês: http://www.scielo.br/ scielo.php?script=sci_serial\&pid=1983-1447\&lng=pt\&nrm=iso 


\section{ABSTRACT}

Quantitative study aimed to identify the enterprising tendency of nurses at a university hospital and to relate them with age, length of work in the hospital and conclusion of the nursing course. This cross-sectional quantitative study was developed in 2010. All 60 nurses from the hospital answered the questionnaire General measure of Enterprising Tendency, which contains five categories. In the creativity category one nurse obtained two points; in need for achievement one nurse totaled 12 points; one nurse obtained two points; in motivation four nurses achieved higher scores; in taking calculated risks, the highest score was 10 points; in autonomy, nine nurses obtained one point each. Individuals aged between 27 and 33 years showed higher enterprising tendencies. Reduced enterprising tendencies were found for nurses aged between 43 and 56 years, graduated more than 17 years ago and with a greater length of work. Actions are necessary to encourage nurses in the age range of enterprising tendency decline and those who graduated longer ago and who have worked for a longer period of time in the hospital.

Descriptors: Nursing. Nursing staff, hospital. Organizational innovation.

Title: Enterprising tendencies of nurses in a university hospital.

\section{INTRODUÇÃO}

O termo "empreendedor" teve sua origem no verbo francês entreprende ou entrepreneur, que significa aquele que está no meio ou no centro de alguma ação. Da mesma maneira, "empreendedor", em sua ascendência inglesa, entrepreneurship, indica posição, grau, relação, estado, qualidade, habilidade, enquanto que na tradução alemã, expressa quem possui ou dirige um negócio o unternehmer, que, em inglês, expressa alguém que é "proprietário-gerente"(1).

Assim, empreendedor é a pessoa que tem a ideia de um produto ou serviço e estabelece uma ação para que essa se torne uma oportunidade de um negócio lucrativo, assumindo os riscos legais e financeiros para $\operatorname{tal}^{(2)}$.

A habilidade de conviver com os possíveis percalços é um requisito imprescindível para o desenvolvimento da prática empreendedora ${ }^{(3)}$, pois o trabalhador empreendedor possui condições de criar soluções com tino inovador, para gerar ações potencialmente lucrativas e modificadoras ${ }^{(4)}$.

O empreendedorismo na enfermagem é evidente desde o século XIX, quando Florence Nightingale atuou na Guerra da Criméia, fundou a Escola de Enfermagem no Hospital Saint Thomas e transformou o cuidado pautado no modelo religioso em um exercício profissional ${ }^{(5)}$. Outros exemplos de figuras empreendedoras na enfermagem são Anna Nery, que atuou no cuidado dos feridos na Guerra do Paraguai e Wanda de Aguiar Horta, a primeira teorista brasileira de enfermagem ${ }^{(6)}$.

As práticas empreendedoras na enfermagem, atualmente, podem ser encontradas em diversos espaços, como no terceiro setor (organizações, associações e fundações que geram bens e serviços públicos à sociedade), nos serviços de consultoria, nas assessorias e atividades organizacionais e nas atividades de ensino e pesquisa, por exemplo, propiciando ao enfermeiro autonomia e reconhecimento $\operatorname{profissional}^{(7)}$.

A cultura empreendedora deve ser estimulada desde a formação universitária em enfermagem, auxiliando na construção de um pensamento inovador, que assume distância do fazer mecanizado, na forma de uma concepção ampliada do conceito de cuidado/saúde, onde novos campos de atuação podem ser explorados e criados, propiciando novas maneiras de intervenção social ${ }^{(8)}$.

O empreendedorismo caracteriza-se, portanto, como catalisador de iniciativas e mudanças, auxiliando enfermeiros a lidar com intempéries comuns a sua profissão, bem como planejar, organizar e desenvolver novas formas de trabalho que aperfeiçoem o seu fazer diário, habilitando-os ao êxito e sucesso por um longo período de tempo em suas carreiras.

Diante do exposto, este estudo teve como objetivos identificar tendências empreendedoras dos enfermeiros de um hospital universitário e relacioná-las com as variáveis idade, tempo de trabalho no hospital e tempo de conclusão do curso de enfermagem.

Esta pesquisa justifica-se pela carência de estudos na área que contemplem a investigação do empreendedorismo no próprio trabalho da enfermagem, tendo como sujeitos os enfermeiros em sua realidade de atuação, pois o empreendedorismo, usualmente, na enfermagem, vem sendo focado na necessidade de fomentá-lo no ensino da graduação, com o intuito de formar profissionais empreendedores. A importância deste estudo está em reconhecer 
enfermeiros potencialmente empreendedores e estimular os demais a desenvolver características empreendedoras que os tornem diferenciados em meio ao simples e comum na profissão.

\section{METODOLOGIA}

Estudo de abordagem quantitativa, transversal contemporâneo desenvolvido em um hospital universitário do sul do Brasil, entre os meses de agosto e outubro de 2010.

Foi constituído pela integralidade dos 60 enfermeiros das diversas unidades assistenciais e administrativas do referido hospital, que concordaram em participar e assinaram o Termo de Consentimento Livre e Esclarecido. A opção pela realização do estudo em um hospital universitário ocorreu por este ser um espaço fértil de pesquisas, propiciando a que os resultados destas sejam mote de discussões e promoção de reflexões, especialmente sobre o empreendedorismo e a importância de incorporá-lo ao processo de trabalho da enfermagem.

Para a coleta dos dados foi utilizado o questionário para medir a Tendência Empreendedora Geral (TEG) desenvolvido na Unidade de Formação Empresarial e Industrial da Durham University Business School-Durham, Inglaterra por C. Johnson e Sally Caird em 1988 e já validado por pesquisadores brasileiros $^{(9)}$. Este questionário visa avaliar características empreendedoras utilizando como parâmetro cinco categorias: (1) necessidade de realização; (2) necessidade de autonomia/independência; (3) criatividade; (4) disposição a riscos; (5) determinação, cada qual com uma pontuação máxima de 12 pontos, à exceção da necessidade de autonomia/independência, que tem a pontuação máxima de 6 pontos.

O TEG é composto de 54 questões e em cada uma das assertivas o respondente assinala A (de acordo) ou D (em desacordo), conforme seu entendimento acerca dos postulados. O cálculo da pontuação é feito da seguinte forma: para cada questão "par" em que o respondente marca A (de acordo) há a soma de um ponto e para cada questão "ímpar" em que marca D (em desacordo) também há a soma de um ponto.

Os dados desta pesquisa, obtidos por meio da aplicação do questionário, foram organizados em uma planilha Excel e analisados através de elementos de Estatística Descritiva. Para análise dos resultados da relação do valor do TEG com as variáveis idade, tempo de trabalho no hospital universitário, tempo de trabalho na mesma unidade ou setor do hospital e tempo de conclusão do curso de enfermagem foram utilizados Diagramas de Dispersão.

Considerando os aspectos éticos da pesquisa, o projeto foi aprovado pelo Comitê de Ética ao qual o hospital universitário está vinculado, através do parecer n. 88/2010.

\section{RESULTADOS}

Os enfermeiros são em 98,33\% mulheres, com idade entre 27 e 56 anos, sendo que $50 \%$ variam entre 32,5 e 49,5 anos. Em relação ao tempo da formação universitária, a amplitude é de 29 anos ( entre 1 e 30 anos ), sendo que $50 \%$ oscilou entre 10 e 22,5 anos. Já no quesito tempo de trabalho no HU, o menor é de 1 mês e o maior de 24 anos, sendo que na mesma unidade/setor, a média de permanência é de 7 anos, com uma variação entre 1 e 16 anos.

Observou-se que os enfermeiros com idade entre 32,5 e 34,5 anos são aqueles que apresentam maior tendência empreendedora. Em contrapartida, os com idade superior a 43 anos demonstram um decréscimo da tendência empreendedora retomando-a e mantendo-a até a faixa dos 56 anos, quando reinicia um novo declínio da pontuação. A Figura 1 ilustra o exposto.

Os enfermeiros com maior tempo de trabalho no hospital mostram menor tendência empreendedora, sendo encontrados altos escores do TEG em enfermeiros com tempo de trabalho entre 4 e 5 anos. Embora não tenha sido objetivo do estudo definir um ponto de corte, há fortes indicativos de que há a partir dos 12 anos de tempo de trabalho no HU há um decréscimo na tendência empreendedora, conforme Figura 2.

Verificou-se que os enfermeiros que obtiveram entre 42 e 44 pontos foram aqueles que possuem um tempo de conclusão de curso que varia de 5 a 11 anos, conforme exposto na Figura 3.

Os dados obtidos indicam que o enfermeiro que alcançou o maior escore na necessidade de realização marcou 12 pontos, possui 33 anos de idade, concluiu o curso de enfermagem em 1999 e trabalha no hospital universitário há 21 meses. Já o enfermeiro que conseguiu a menor nota obteve 5 pontos, possui 52 anos de idade, é formado desde 1985 e trabalha no hospital universitário há 22 anos. 


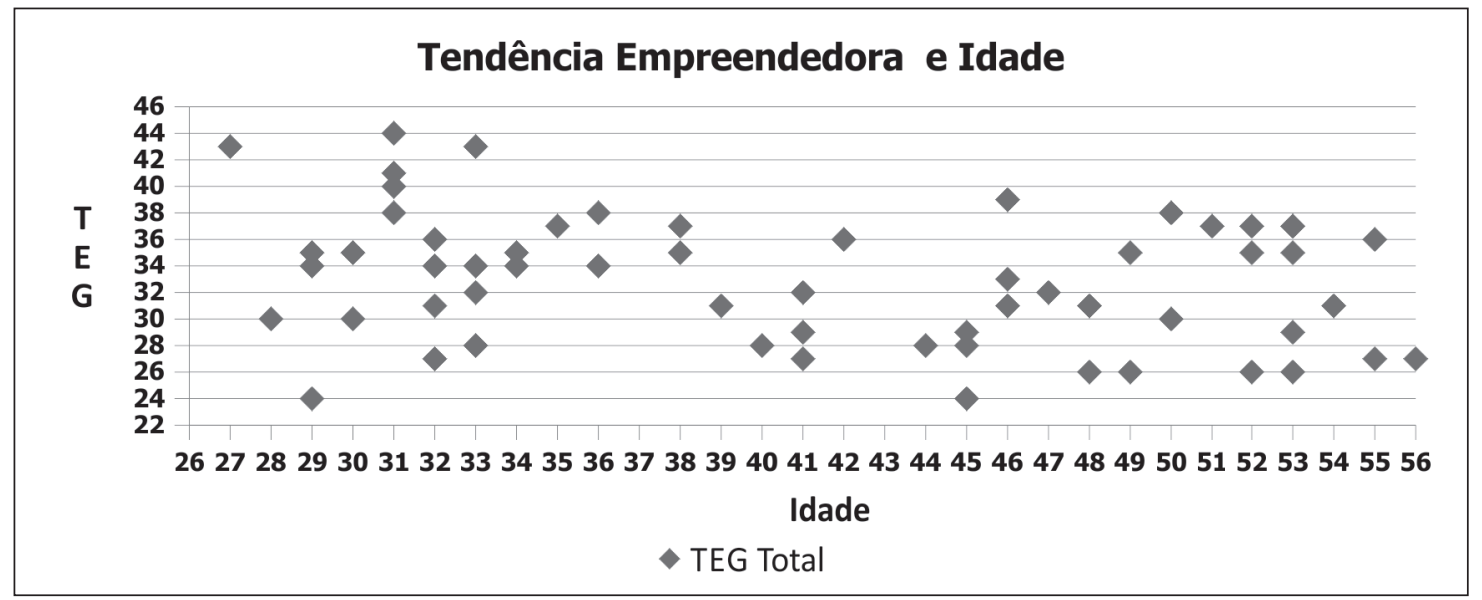

Figura 1 - Relação entre a Tendência Empreendedora e a Idade dos enfermeiros do HU. Rio Grande, RS, 2010.

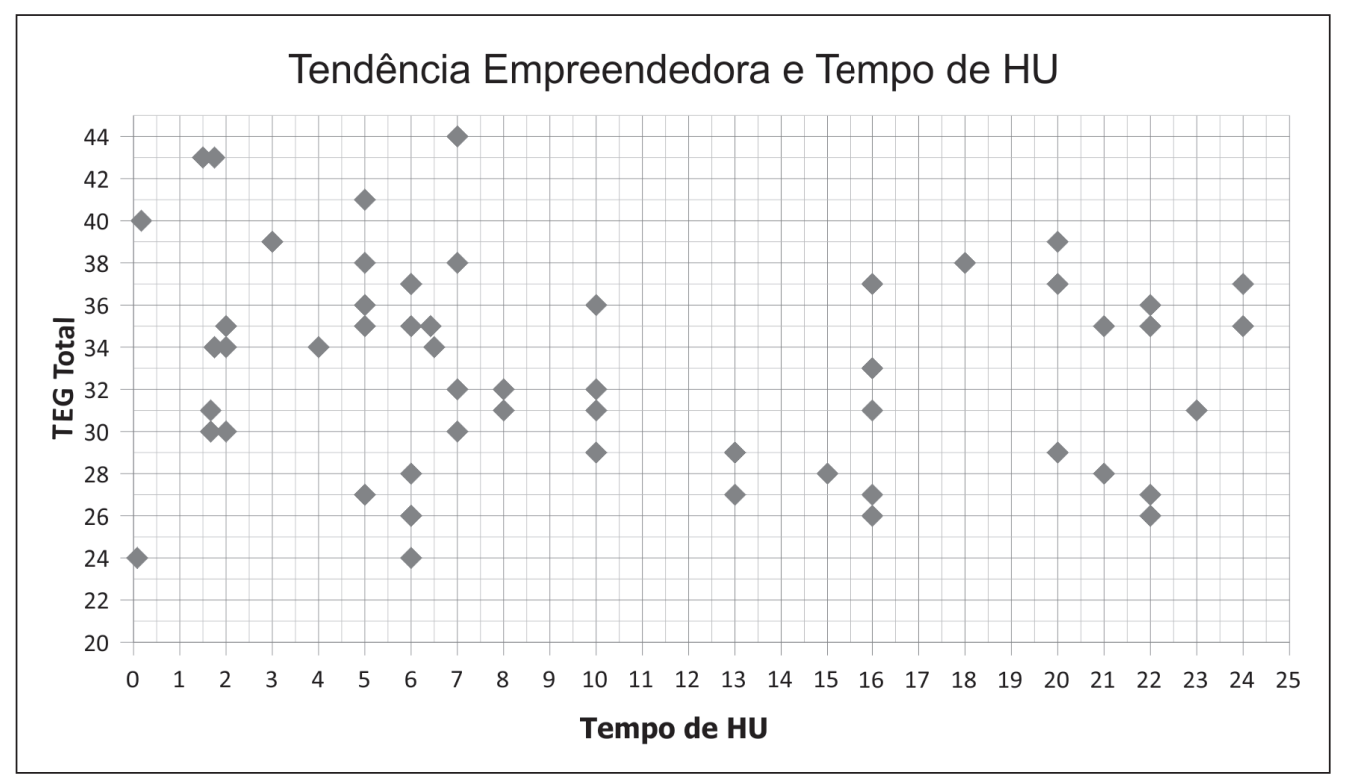

Figura 2 - Relação entre a Tendência Empreendedora e o Tempo de trabalho no HU. Rio Grande, RS, 2010.

O enfermeiro de maior escore no quesito necessidade de autonomia/independência alcançou 6 pontos, possui 32 anos de idade, concluiu o curso de enfermagem em 2002 e trabalha no HU há quatro anos. Nesta categoria nove enfermeiros alcançaram a menor pontuação com um ponto, as idades variaram entre 28 e 53 anos de idade, o tempo de conclusão do curso oscilou entre 1982 e 2007 e o tempo de trabalho no HU permaneceu entre 20 meses e 16 anos.

Em tendência criativa, a maior pontuação foi de 12 pontos e o enfermeiro possui 29 anos, é formado desde 2004 e trabalha no HU há dois anos.
O menor escore foi de 2 pontos, de um enfermeiro com 29 anos de idade, formado desde 2008, que trabalha há um mês no HU.

Em Riscos Calculados/Moderados, o enfermeiro que atingiu maior nota alcançou 10 pontos, possui 34 anos de idade, é formado desde 1998 e trabalha no HU há vinte e um meses. O enfermeiro com menor pontuação teve 1 ponto, possui 55 anos de idade, é formado desde 1980 e trabalha no HU há vinte e dois anos.

Em Impulso e Determinação, quatro enfermeiros alcançaram 12 pontos. A idade variou entre 41 


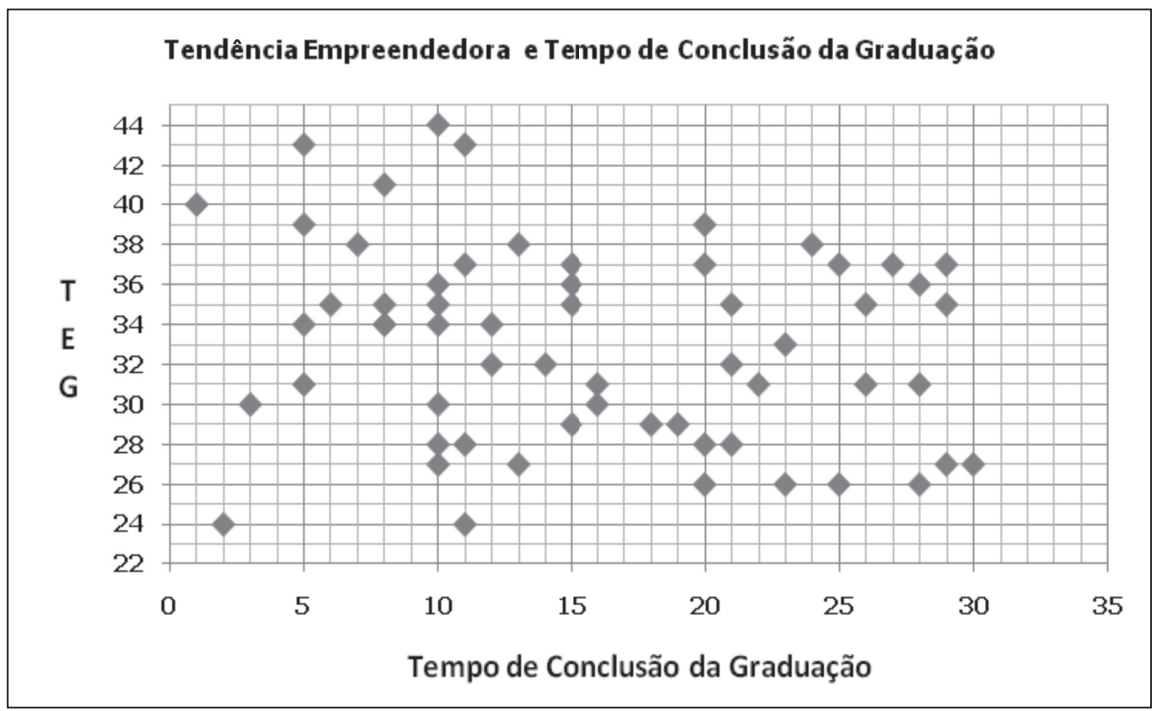

Figura 3 - Relação entre a Tendência Empreendedora e o Tempo de Conclusão de Curso dos Enfermeiros do HU. Rio Grande, RS, 2010.

e 55 anos, a formação entre 1980 e 1995 e o tempo de trabalho no HU oscilou entre 16 e 22 anos. O enfermeiro com menor escore totalizou 6 pontos, possui 28 anos de idade, concluiu sua formação em 2008 e atua no HU há um mês.

\section{DISCUSSÃO}

A relação entre idade, tempo de formação e os resultados obtidos por meio do questionário evidenciam dois aspectos: o decréscimo da tendência empreendedora dos enfermeiros com idade superior a 43 e até 56 anos e daqueles com mais de 17 anos de formados.

A combinação entre longo tempo de formação e baixa tendência empreendedora pode estar vinculada aos resquícios de um perfil de formação de enfermeiros que ocorria até os anos 80 do século $\mathrm{XX}$, o qual era mais voltado aos aspectos vocacionais do cuidado e ao cumprimento de normas organizacionais, em detrimento das ações mais empreendedoras $^{(10)}$.

Paralelamente, o aumento da idade associada ao decréscimo do TEG tem a possibilidade de estar vinculado ao desgaste proporcionado pelo trabalho da enfermagem, muitas vezes exaustivo e complexo, sendo as longas jornadas e o estresse determinantes à diminuição das tendências empreendedoras dos enfermeiros.

A própria legislação do Conselho Federal de Enfermagem para dimensionamento de pessoal de enfermagem parece reforçar a diminuição da produtividade dos enfermeiros com mais de 50 anos, quando recomenda, no Art. $9^{\circ}$ que "O quadro de profissionais de enfermagem da unidade de internação composto por $60 \%$ ou mais de pessoas com idade superior a 50 (cinqüenta) anos, deve ser acrescido de $10 \%$ ao IST"(11).

No mesmo rumo, a correlação entre tempo de trabalho no HU com o total da pontuação obtida por meio do TEG identifica que os enfermeiros com maior tempo de trabalho mostram menor tendência empreendedora, sendo que os maiores valores foram obtidos pelos enfermeiros com tempo de trabalho entre 4 e 5 anos. Isto pode se justificar pelas mesmas alegações expostas acima e encontra eco no estudo de Wandemberg( ${ }^{(12)}$ intitulado "Empreender na era do fim do emprego" em que o autor reitera que, permanecer durante um longo período desempenhando a mesma função, em uma mesma instituição, torna-se um fator prejudicial em relação ao empreendedorismo.

Em relação à dimensão "necessidade de realização" verificou-se que o sujeito com maior pontuação está a pouco tempo no hospital universitário, em oposição à enfermeira com menor escore que está há 22 anos na instituição, tempo proporcional também em relação à formatura de ambas. A “necessidade de realização” está vinculada à satisfação pessoal e é a necessidade que o empreendedor deve alcançar para obter sucesso em seu empreendimento ${ }^{(13)}$. Ao obter uma pontuação considerada elevada, a 
enfermeira que marcou 12 pontos pode apresentar as seguintes características: visão voltada para o futuro, auto-suficiência, otimismo, orientação para tarefas e resultados, energia, confiança em si mesmo, persistência, determinação e dedicação para concluir suas funções ${ }^{(14)}$, condições essenciais para o desempenho satisfatório do trabalho da enfermagem. Esta necessidade é fundamental para que os enfermeiros encontrem sentido na profissão e empreendam ações no seu trabalho de modo que atinjam tanto seus objetivos pessoais como aqueles erigidos pela organização onde trabalham.

O enfermeiro que obteve 12 pontos na categoria "tendência criativa" pode demonstrar qualidades como imaginação e inovação, versatilidade e curiosidade, produção de muitas ideias, intuição, gosto por novos desafios, novidade e mudança ${ }^{(15)}$, estratégias e táticas necessárias para driblar dificuldades e obstáculos advindos do trabalho hospitalar. Um estudo reitera a importância do uso da criatividade pelos trabalhadores da enfermagem, uma vez que esta pode auxiliar na realização de adaptações e improvisações, a fim de que o cuidado seja efetivo e as tarefa sejam cumprida ${ }^{(16)}$.

No item “impulso e determinação" quatro enfermeiros obtiveram pontuação máxima (12 pontos). Uma pontuação alta nesta seção significa que a pessoa tende a aproveitar as oportunidades, não aceitar a predestinação, fazer a sua própria sorte, controlar seu destino, ser autoconfiante, equilibrar resultado com esforço e mostrar considerável determinação ${ }^{(14)}$

É importante realçar que um dos enfermeiros com 12 pontos é enfermeiro folguista, isto é, aquela que desenvolve suas atividades nos mais diferentes cenários do HU no noturno, o que a faz enfrentar adversidades e situações imprevistas a cada plantão, uma vez que não tem uma equipe de trabalho de referência, nem tampouco identidade com uma unidade ou setor. Da mesma maneira, outro enfermeiro com a pontuação máxima exerce suas funções na área administrativa, lidando diariamente com toda sorte de problemas do pessoal de enfermagem. Estas duas situações podem indicar que a necessidade de superação no trabalho pode fazer com que os enfermeiros potencializem suas características empreendedoras de "impulso e determinação".

Quanto ao atributo "necessidade de autonomia/independência" verificou-se a menor pontuação de todos os enfermeiros, indicando, talvez, que os enfermeiros do hospital universitário ainda estejam vinculados à idéia de que os médicos e ad- ministradores nos espaços hospitalares ainda são os que controlam estes espaços, determinando as políticas e os objetivos organizacionais ${ }^{(17)}$.

Neste pensamento, os profissionais de enfermagem estão colocados em uma situação em que os limites de sua autonomia são desenhados pela autonomia do trabalho médico, pois mesmo sendo fundamental, seu trabalho ainda é, em muitos espaços, auxiliar ${ }^{(18)}$

Esta afirmativa, porém, pode ser contraposta uma vez que a coordenação do hospital é de fato da enfermagem, mesmo que exista uma relação paradoxal, pois quem coordena o cuidado é o pólo dominado nesta relação de dominação ${ }^{(19)}$. Estudos como "A autonomia profissional do enfermeiro" assevera o exposto e argumenta que a enfermagem possui amplos espaços de exercício de autonomia e de poder decisório, tanto em seu processo de trabalho como na administração das organizações de saúde ${ }^{(20)}$.

A característica "riscos calculados/moderados", cujos resultados variaram de 1 a 10 pontos, define que o empreendedor deve buscar situações desafiadoras que possuam riscos calculados e também estar constantemente avaliando os riscos ligados ao seu empreendimento, estando suas recompensas vinculadas a esses riscos ${ }^{(19)}$. A baixa pontuação pode estar ligada à questão de segurança no emprego, já que a maioria dos enfermeiros está ligada a Regime Jurídico Único, o que lhes oferece estabilidade no emprego.

Assim, quanto a este quesito, surge o questionamento: - A estabilidade estaria provocando uma diminuição da tendência empreendedora dos enfermeiros do HU? - Haveria diferenciação neste item se a pesquisa fosse realizada com enfermeiros de hospitais privados em que não há estabilidade?

$\mathrm{Na}$ outra ponta desta discussão acerca da característica "riscos calculados/moderados" está, novamente, a alta pontuação alcançada pelo enfermeiro folguista, relacionando, possivelmente sua função com esta característica, uma vez que a cada jornada ela, inevitavelmente, encontra uma nova e desafiadora realidade, muitas vezes, inesperada.

\section{CONCLUSÕES}

O objetivo deste estudo foi de identificar as tendências empreendedoras dos enfermeiros de um hospital universitário e relacioná-las às variáveis idade, tempo de trabalho no hospital universitário e tempo de conclusão do curso. 
As evidências e discussões possibilitaram o entendimento do fenômeno do empreendedorismo na realidade dos enfermeiros do hospital, mesmo que a pesquisa tenha retratado um momento específico daquele espaço e sujeitos. Tem-se a clareza de que outros estudos, que envolvam, concomitantemente, subjetividades da cultura e do clima organizacional, também sejam pertinentes e complementares para maior imersão nesse universo.

Entretanto, por ora, faz-se necessário que o HU promova ações no sentido de estimular aqueles enfermeiros que estão na faixa etária detectada no estudo como em declínio de tendência empreendedora, bem como aqueles com maior tempo de formação e de trabalho no hospital, como forma de revigorar seu trabalho e, com isso, promover direta e indiretamente, a qualidade da assistência de enfermagem. Também é imprescindível o desenvolvimento de características profissionais e pessoais que caminhem para o desempenho empreendedor dos enfermeiros no rumo da realização, do espírito criativo, da determinação e da autonomia profissional. Discussões internas sobre a questão dos riscos calculados e o seu papel para o melhor aproveitamento do trabalho empreendedor também devem ser incentivadas e promovidas.

Nestas iniciativas é importante a participação dos enfermeiros que foram detectados como potencialmente empreendedores, para que atuem como multiplicadores, estimulando os demais a ampliar características empreendedoras para promover e qualificar o trabalho da enfermagem.

Ao findar este estudo, verifica-se que existe a necessidade de encorajar o empreendedorismo em enfermeiros em todos os momentos da trajetória profissional, para que os mesmos se tornem diferenciados em meio ao simples e ao comum na profissão e tenham persistência, tenacidade e determinação em permanecerem aguçados para o novo, atuando com autonomia e buscando sua realização no trabalho e em suas vidas pessoais.

\section{REFERÊNCIAS}

1 Drucker PF. As novas realidades. $4^{\mathrm{a}}$ ed. São Paulo: Cengage Learning; 2003.

2 Oliveira M. Valeu: passos na trajetória de um empreendedor. São Paulo: Nobel; 1995.

3 Roncon PF, Munhoz S. Estudantes de enfermagem têm perfil empreendedor? Rev Bras Enferm. 2009;62(5):695-700.
4 Drucker PF. Inovação e espírito empreendedor: prática e princípios. São Paulo: Cengage Learning; 2008.

5 Padilha MICS, Borenstein MS, Santos I. Enfermagem: história de uma profissão. São Caetano do Sul: Difusão; 2011.

6 Conselho Regional de Enfermagem (COREN-SP). História da enfermagem: as práticas de saúde ao longo da historia e o desenvolvimento das práticas de enfermagem [Internet]. [2000] [citado 2010 Nov 19]. Disponível em: http://inter.coren-sp.gov. $\mathrm{br} /$ node/382 1 .

7 Erdmann AL, Backes SD, Alves A, Albino AT, Farias F, Guerini IC, et al. Formando empreendedores na enfermagem: promovendo competências e aptidões sócio-políticas. Enferm Glob. 2009;16:1-10.

8 Backes DS, Erdmann AL. Formação do enfermeiro pelo olhar do empreendedorismo social. Rev Gaúcha Enferm. 2009;30(2):242-8.

9 Gaião BFDS, Silva TA, Queiroz CTAP. Diagnóstico da tendência empreendedora através do modelo de Durham: um estudo de caso no setor educacional. Qu@litas Rev Eletr. 2009; 8(3).

10 Lunardi Filho WD. O mito da subalternidade do trabalho da enfermagem à medicina. Pelotas: Universitária/UFPel; 2000.

11 Conselho Federal de Enfermagem (COFEN). Resolução n. 293/2004: fixa e estabelece parâmetros para dimensionamento do quadro de profissionais de enfermagem nas instituições de saúde [Internet]. Rio de Janeiro; 2004 [citado 2010 Nov 20]. Disponível: http://corensp.org.br/072005/legislacoes/legislacoes_busca.php?leg_id=10105\&text.

12 Wandemberg A. Empreender na era do fim do emprego [Internet]. 2008 [citado 2010 Nov 20]. Disponível em: http://artigos.netsaber.com.br/ resumo_artigo_6591/artigo_sobre_empreender_na_ era_do_fim_do_emprego. Acesso em: 19 Nov. 2010. Acessado em: 20 nov 2010.

13 Uriarte LR. Tendência empreendedora das profissões. In: Anais do $1^{\circ}$ Encontro Nacional de Empreendedorismo; 1999; Florianópolis, Brasil. Florianópolis: UFSC; 1999 .

14. Andujar AM. Modelo de qualidade de vida dentro dos domínios bio-psicosocial para aposentados [tese]. 
Florianópolis (SC): Universidade Federal de Santa Catarina; 2006.

15 Bernardi LA. Manual de empreendedorismo e gestão. $2^{\text {a }}$ ed. São Paulo: Atlas; 2007.

16 Souza NVDO, Santos DM, Anunciação CT, Thiengo PCS. O trabalho da Enfermagem e a criatividade: adaptações e improvisações hospitalares. Rev Enferm UERJ. 2009;17(3):356-61.

17 Carapinheiro G. Saberes e poderes no hospital: uma sociologia dos serviços hospitalares. Porto: Afrontamento; 2005.
18 Marques GQ, Lima MADS. Organização tecnológica do trabalho em um pronto atendimento e a autonomia do trabalhador de enfermagem. Rev Esc Enferm USP [Internet]. 2008. [citado 2013 Mai 09];42(1):41-7. Disponível em: http://www.scielo.br/scielo.php?script=sci arttext\&pid=So080-62342008000100006\&lng=en http://dx.doi.org/10.1590/S0080-62342008000100006

19 Merhy EE, Cecílio LCO. Algumas reflexões sobre o singular processo de coordenação dos hospitais. Saúde Debate. 2003;22(64):110-12.

20 Lunardi Filho WD. A autonomia profissional do enfermeiro. Enferm Brasil 2008;6(3):1.

Recebido em: 28.05.2012

Aprovado em: 27.05.2013

\section{Author's address}

Helena Heidtmann Vaghetti

Rua Dr. Nascimento, 292, Centro

96200-300, Rio Grande, RS

E-mail:vaghetti@vetorial.net 